\title{
Stabilization, Lyapunov functions, and dissipation
}

\author{
P. Rapisarda ${ }^{\mathrm{a}, *}$, C. Kojima ${ }^{\mathrm{b}, 1}$ \\ a Signals, Images, Systems Research Group, School of Electronics and Computer Science, University of Southampton, Southampton SO17 1BJ, United Kingdom

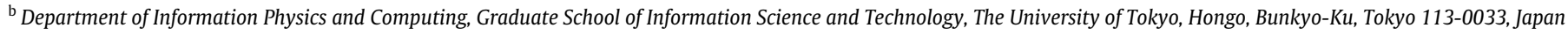

\section{A R T I C L E I N F O}

\section{Article history:}

Received 19 October 2009

Received in revised form

12 July 2010

Accepted 20 September 2010

Available online 27 October 2010

\section{Keywords:}

Stabilization

(Half-line) dissipativity

Linear systems

Interconnection

Lyapunov functions

\begin{abstract}
A B S T R A C T
For linear time-invariant systems, any stabilizing controller for a given plant can be associated with a supply rate with respect to which the plant in open-loop is half-line dissipative. We also prove the equivalence between the stability of the interconnection of two systems and their half-line dissipativity with respect to a supply rate and its negative.
\end{abstract}

(c) 2010 Elsevier B.V. All rights reserved.

\section{Introduction}

In this paper, we show that interconnecting a plant with a stabilizing controller can be interpreted as imposing on the plant the requirement that its trajectories are dissipative with respect to a dynamic supply rate induced by the controller. This supply rate is represented by a two-variable polynomial matrix which can be expressed in terms of the polynomial matrix representing the controller, or equivalently of the matrices inducing a Lyapunov function and its derivative for the closed-loop behavior.

To the best of the authors' knowledge, and to their great surprise, this result is not to be found in the literature, although the relation between Lyapunov functions, stabilizing controllers, and solutions of the Riccati equation (and consequently, storage functions and dissipativity) has been studied by many authors. Identifying stabilization and dissipation, a result eminently reasonable if not altogether commonsensical, is also important for the following reason. It is well known that, in the nonlinear setting, one way of stabilizing a system is to interconnect to it a passivating controller (see [1-4]): passivation is sufficient in order to achieve closed-loop stability. However, Theorem 6 of this paper states that, in the linear setting, stabilizing a plant amounts to imposing dissipation on it: passivation with respect to a suitable supply rate is necessary in order to achieve closed-loop stability.

\footnotetext{
* Corresponding author. Tel.: +44 0238059 3367; fax: +44 02380594498. E-mail addresses: pr3@ecs.soton.ac.uk (P. Rapisarda), chiaki_kojima@ipc.i.u-tokyo.ac.jp (C. Kojima).

1 Tel.: +8135841 6890; fax: +81358416890.
}

The second result presented in this paper is that stability of the interconnection of two higher-order linear differential systems is equivalent to the existence of a quadratic functional $Q_{\Phi}$ such that one of the systems is $Q_{\Phi}$-half-line dissipative, and the other one is $-Q_{\Phi}$-half-line dissipative. Moreover, the dissipation function for $Q_{\Phi}$ (respectively, $-Q_{\Phi}$ ) can be chosen to be strictly positive along the trajectories of the interconnected system. Furthermore, we show how to compute such a supply rate $Q_{\Phi}$ starting from a representation of the interconnected system. While preparing this paper, the authors have been made aware of the recent results of Takaba on the same topic; see [5].

In this article, we deal with plants and controllers described by systems of higher-order linear differential equations, and we consider control as interconnection (see [6]), rather than adopting the usual "intelligent control" framework. There are good reasons for this level of generality: on the one hand, modeling a physical system from first principles hardly ever results in a statespace description, which usually needs to be constructed from the set of higher-order differential equations (possibly with static constraints among the variables) describing the model (see [7]). On the other hand, many physical and engineering situations put into question the classical point of view of the controller as a signal processor transforming inputs in outputs (see for example the analysis of a simple door-closing mechanism illustrated in [6]).

The behavioral framework provides the right algebraic tools and theoretical concepts for dealing with higher-order representations; among these are the calculus of behaviors based on one-variable polynomial algebra (see [8]), and the calculus of functionals of the system variables and their derivatives based on 
two-variable polynomial algebra (see [9]). Moreover, the concept of control as interconnection is central to the behavioral framework. In this paper, these concepts and tools will be put to strenuous use. In Section 2, we briefly review the definitions and concepts necessary in the rest of the paper. Section 3 contains our result equating dissipativity and stability in the linear case. We analyze the relation between stability of interconnections and dissipativity in Section 4. Section 5 draws the conclusions.

Notation. The space of $n$-dimensional real vectors is denoted by $\mathbb{R}^{\mathrm{n}}$, and the space of $\mathrm{m} \times \mathrm{n}$ real matrices by $\mathbb{R}^{\mathrm{m} \times \mathrm{n}}$. If one of the dimensions is not specified, a bullet $\bullet$ is used. Given two matrices $A$ and $B$ with the same number of columns, we denote with $\operatorname{col}(A, B)$ the matrix obtained by stacking $A$ over $B$. The ring of polynomials with real coefficients in the indeterminate $\xi$ is denoted by $\mathbb{R}[\xi]$; the ring of polynomials with real coefficients in the indeterminates $\zeta$ and $\eta$ is denoted by $\mathbb{R}[\zeta, \eta]$. The set of $\mathrm{n} \times \mathrm{m}$ polynomial matrices in $\xi$ is denoted by $\mathbb{R}^{\mathrm{n} \times \mathrm{m}}[\xi]$, and that consisting of all $\mathrm{n} \times \mathrm{m}$ polynomial matrices in $\zeta$ and $\eta$ by $\mathbb{R}^{\mathrm{n} \times \mathrm{m}}[\zeta, \eta]$. We denote with $\mathfrak{C}^{\infty}\left(\mathbb{R}, \mathbb{R}^{\mathrm{w}}\right)$ the set of infinitely often differentiable functions from $\mathbb{R}$ to $\mathbb{R}^{\mathrm{w}}$. The set of infinitely differentiable trajectories with compact support is denoted with $\mathfrak{D}\left(\mathbb{R}, \mathbb{R}^{\mathrm{W}}\right)$.

\section{Background material}

\subsection{Linear differential behaviors}

A linear differential behavior is a linear subspace $\mathfrak{B} \subseteq \mathfrak{C}^{\infty}\left(\mathbb{R}, \mathbb{R}^{\mathrm{w}}\right)$ consisting of all solutions of a system of linear, constant-coefficient differential equations in w variables. We denote with $\mathfrak{L}^{\mathrm{w}}$ the set consisting of all linear differential behavior with w variables. $\mathfrak{B} \in$ $\mathfrak{L}^{\mathrm{W}}$ can always be represented as

$R\left(\frac{\mathrm{d}}{\mathrm{d} t}\right) w=0$

where $R \in \mathbb{R}^{\bullet \times w}[\xi]$. Eq. (1) is called a kernel representation of $\mathfrak{B}$, and $w$ is called the manifest variable of $\mathfrak{B}$.

Let $R \in \mathbb{R}^{\bullet \times \mathrm{w}}[\xi], M \in \mathbb{R}^{\bullet \times \mathrm{m}}[\xi]$, and consider the equations

$R\left(\frac{\mathrm{d}}{\mathrm{d} t}\right) w=M\left(\frac{\mathrm{d}}{\mathrm{d} t}\right) \ell$.

Eq. (2) is called a hybrid representation of the external behavior

$\mathfrak{B}:=\left\{w \in \mathfrak{C}^{\infty}\left(\mathbb{R}, \mathbb{R}^{\mathrm{w}}\right) \mid \exists \ell \in \mathfrak{C}^{\infty}\left(\mathbb{R}, \mathbb{R}^{\mathrm{m}}\right)\right.$ s.t. Eq. (2) holds $\}$.

The set $\mathfrak{B}_{\text {full }}:=\left\{(w, \ell) \in \mathfrak{C}^{\infty}\left(\mathbb{R}, \mathbb{R}^{\mathrm{w}+\mathrm{m}}\right) \mid\right.$ Eq. (2) holds $\}$ is called the full behavior of (2).

Associated with a system in $\mathfrak{L}^{\mathrm{w}}$ there are a number of integer invariants (see [8]). In the following, we will refer frequently to $\mathrm{p}(\mathfrak{B})$, the number of output variables of $\mathfrak{B}$, also called the output cardinality of the behavior $\mathfrak{B}$; and $\mathrm{m}(\mathfrak{B})$ the number of input variables of $\mathfrak{B}$, also called the input cardinality of the behavior $\mathfrak{B}$. These numbers satisfy $\mathrm{p}(\mathfrak{B})+\mathrm{m}(\mathfrak{B})=\mathrm{w}$.

The notion of autonomous behavior plays an important role in this paper; we refer the reader to $\mathrm{Ch}$. 3 of [8] for the definition, and quote here only the following result.

Proposition 1. Let $\mathfrak{B} \in \mathfrak{L}^{\mathrm{w}}$. The following statements are equivalent:

1. $\mathfrak{B}$ is autonomous;

2. $\mathrm{m}(\mathfrak{B})=0$;

3. $\mathrm{p}(\mathfrak{B})=\mathrm{w}$;

4. There exists $R \in \mathbb{R}^{\mathrm{w} \times \mathrm{w}}[\xi]$ such that $\operatorname{det}(R) \neq 0$ and $\mathfrak{B}=\operatorname{ker} R$ $\left(\frac{\mathrm{d}}{\mathrm{d} t}\right)$.

\subsection{Quadratic differential forms}

Let $\Phi \in \mathbb{R}^{\mathrm{w} \times \mathrm{w}}[\zeta, \eta]$; then $\Phi(\zeta, \eta)=\sum_{h, k=0}^{N} \Phi_{h, k} \zeta^{h} \eta^{k}$, where $\Phi_{h, k} \in \mathbb{R}^{\mathrm{w} \times \mathrm{w}}$ and $N \in \mathbb{N} \cup\{0\} . \Phi(\zeta, \eta)$ induces a quadratic functional $Q_{\Phi}$, called a quadratic differential form $(Q D F)$, as follows:

$Q_{\Phi}: \mathfrak{C}^{\infty}\left(\mathbb{R}, \mathbb{R}^{\mathrm{W}}\right) \longrightarrow \mathfrak{C}^{\infty}(\mathbb{R}, \mathbb{R})$,

$Q_{\Phi}(w):=\sum_{h, k=0}^{N}\left(\frac{\mathrm{d}^{h} w}{\mathrm{~d} t^{h}}\right)^{T} \Phi_{h, k} \frac{\mathrm{d}^{k} w}{\mathrm{~d} t^{k}}$.

Without loss of generality, in the following when dealing with QDFs we assume that $\Phi(\zeta, \eta)$ is symmetric, i.e. $\Phi(\zeta, \eta)=\Phi$ $(\eta, \zeta)^{\top}$. We denote the set of all symmetric $\mathrm{w} \times \mathrm{w}$ two-variable polynomial matrices with $\mathbb{R}_{S}^{\mathrm{W} \times \mathrm{w}}[\zeta, \eta]$.

A QDF $Q_{\Phi}$ is called nonnegative, denoted $Q_{\Phi} \geq 0$, if $Q_{\Phi}(w) \geq 0$ for all $w \in \mathfrak{C}^{\infty}\left(\mathbb{R}, \mathbb{R}^{\mathrm{w}}\right)$, and positive, denoted $Q_{\Phi}>0$, if $Q_{\Phi} \geq 0$ and $\left[Q_{\Phi}(w)=0\right] \Longrightarrow[w=0]$. Let $\mathfrak{B} \in \mathfrak{L}^{\mathrm{w}}$; then $Q_{\Phi}$ is called nonnegative along $\mathfrak{B}$, denoted $Q_{\Phi} \stackrel{\mathfrak{B}}{\geq} 0$, if $Q_{\Phi}(w) \geq 0$ for all $w \in \mathfrak{B}$. The concept of a $\mathrm{QDF}$ positive along $\mathfrak{B}$, denoted $\stackrel{\mathfrak{B}}{>} 0$, follows immediately.

We say that $Q_{\Psi}$ is the derivative of $Q_{\Phi}$ if $\left(\frac{\mathrm{d}}{\mathrm{d} t} Q_{\Phi}\right)(w):=\frac{\mathrm{d}}{\mathrm{d} t}$ $\left(Q_{\Phi}(w)\right)=Q_{\Psi}(w)$ for all $w \in \mathfrak{C}^{\infty}\left(\mathbb{R}, \mathbb{R}^{w}\right)$. In polynomial terms, this relationship is equivalently expressed (see p. 1710 of [9]) as

$\left[Q_{\Psi}=\frac{\mathrm{d}}{\mathrm{d} t} Q_{\Phi}\right] \Longleftrightarrow[\Psi(\zeta, \eta)=(\zeta+\eta) \Phi(\zeta, \eta)]$.

Let $\mathfrak{B} \in \mathfrak{L}^{\mathrm{w}}$; two QDFs $Q_{\Phi_{1}}$ and $Q_{\Phi_{2}}$ are called equivalent on $\mathfrak{B}$ (briefly, $\mathfrak{B}$-equivalent), denoted $Q_{\Phi_{1}} \stackrel{\mathfrak{B}}{=} Q_{\Phi_{2}}$, if $Q_{\Phi_{1}}(w)=Q_{\Phi_{2}}(w)$ for all $w \in \mathfrak{B}$. The following result (see Proposition 3.2 of [9]) holds true.

Proposition 2. Let $\mathfrak{B}=\operatorname{ker} R\left(\frac{\mathrm{d}}{\mathrm{d} t}\right)$; then $Q_{\Phi_{1}} \stackrel{\mathfrak{B}}{=} Q_{\Phi_{2}}$ if and only if there exists $F \in \mathbb{R}^{\bullet \bullet} \cdot[\zeta, \eta]$ such that

$\Phi_{1}(\zeta, \eta)=\Phi_{2}(\zeta, \eta)+R(\zeta)^{\top} F(\zeta, \eta)+F(\eta, \zeta)^{\top} R(\eta)$

If (4) holds, in the following we also write $\Phi_{1}(\zeta, \eta) \stackrel{\mathfrak{B}}{=} \Phi_{2}(\zeta, \eta)$. If $\mathfrak{B} \in \mathfrak{L}^{\mathrm{W}}$ is autonomous, then each equivalence class of QDFs under $\stackrel{\mathfrak{B}}{=}$ admits a canonical representative, which we now introduce. Let $\mathfrak{B}=\operatorname{ker} R\left(\frac{\mathrm{d}}{\mathrm{dt}}\right)$ with $R \in \mathbb{R}^{\mathrm{W} \times \mathrm{w}}[\xi]$ such that $\operatorname{det}(R) \neq 0$; it can be proved (see Proposition 4.9 of [9]) that, if $\Phi \in \mathbb{R}_{S}^{\mathrm{W} \times \mathrm{w}}[\zeta, \eta]$, then there exists exactly one $\Phi^{\prime} \in \mathbb{R}_{S}^{\mathrm{W} \times \mathrm{w}}[\zeta, \eta]$ such that $\Phi^{\prime} \stackrel{\mathfrak{B}}{=} \Phi$ and $\left(R(\zeta)^{T}\right)^{-1} \Phi(\zeta, \eta)(R(\eta))^{-1}$ is a matrix of strictly proper two-variable rational functions; $\Phi^{\prime}$ is the $R$ canonical representative of $\Phi$.

\subsection{Dissipative behaviors}

Let $\Phi \in \mathbb{R}_{s}^{\mathrm{w} \times \mathrm{w}}[\zeta, \eta]$ and let $\mathfrak{B} \in \mathfrak{L}^{\mathrm{w}}$ be controllable (see Ch. 5 of [8] for the definition); then $\mathfrak{B}$ is said to be dissipative with respect to the supply rate $Q_{\Phi}$ if

$\int_{-\infty}^{+\infty} Q_{\Phi}(w) \mathrm{d} t \geq 0$ for all $w \in \mathfrak{B} \cap \mathfrak{D}\left(\mathbb{R}, \mathbb{R}^{\mathrm{w}}\right) ;$

note that a controllable behavior always contains compact-support trajectories. If it holds that $\int_{0}^{+\infty} Q_{\Phi}(w) \mathrm{d} t \geq 0$ for all $w \in \mathfrak{B} \cap$ $\mathfrak{D}\left(\mathbb{R}, \mathbb{R}^{\mathrm{w}}\right)$, then $\mathfrak{B}$ is said to be $\mathbb{R}^{+}$-half-line dissipative with respect to the supply rate $Q_{\Phi}$.

A QDF $Q_{\Psi}$ is a storage function for $\mathfrak{B}$ with respect to the supply rate $Q_{\Phi}$ if there holds the dissipation inequality

$\frac{\mathrm{d}}{\mathrm{d} t} Q_{\Psi}(w) \leq Q_{\Phi}(w)$ for all $w \in \mathfrak{B} \cap \mathfrak{D}\left(\mathbb{R}, \mathbb{R}^{\mathrm{W}}\right)$. 
A QDF $Q_{\Delta}$ is a dissipation function for $\mathfrak{B}$ with respect to $Q_{\Phi}$ if

$Q_{\Delta} \stackrel{\mathfrak{B}}{\geq} 0$ and $\int_{-\infty}^{+\infty} Q_{\Phi}(w) \mathrm{d} t=\int_{-\infty}^{+\infty} Q_{\Delta}(w) \mathrm{d} t$

for all $w \in \mathfrak{B} \cap \mathfrak{D}\left(\mathbb{R}, \mathbb{R}^{\mathrm{W}}\right)$.

Storage functions, supply rates, and dissipation functions are related as follows (see Theorem 4.3 of [10]).

Proposition 3. Let $\Phi \in \mathbb{R}_{s}^{\mathrm{W} \times \mathrm{w}}[\zeta, \eta]$ and $\mathfrak{B}=\operatorname{Ker} R\left(\frac{\mathrm{d}}{\mathrm{d} t}\right) \in \mathfrak{L}^{\mathrm{w}}$ controllable. The following statements are equivalent:

1. $\mathfrak{B}$ is dissipative with respect to the supply rate $Q_{\Phi}$;

2. There exists a storage function for $\mathfrak{B}$ with respect to the supply rate $Q_{\Phi}$;

3. There exists a dissipation function for $\mathfrak{B}$ with respect to the supply rate $Q_{\Phi}$.

Given a supply rate $Q_{\Phi}$, there exists a one-to-one relation between storage functions $Q_{\Psi}$ and dissipation functions $Q_{\Delta}$, defined by the dissipation equality

$\frac{\mathrm{d}}{\mathrm{d} t} Q_{\Psi} \stackrel{\mathfrak{B}}{=} Q_{\Phi}-Q_{\Delta}$,

or equivalently $(\zeta+\eta) \Psi(\zeta, \eta)+\Delta(\zeta, \eta)=\Phi(\zeta, \eta)+R(\zeta)^{\top}$ $F(\zeta, \eta)+F(\eta, \zeta)^{\top} R(\eta)$ for some $F \in \mathbb{R}^{\bullet \times \mathrm{w}}[\zeta, \eta]$.

\subsection{Lyapunov theory}

A behavior $\mathfrak{B} \in \mathfrak{L}^{\mathrm{w}}$ is called asymptotically stable if

$[w \in \mathfrak{B}] \Longrightarrow\left[\lim _{t \rightarrow \infty} w(t)=0\right]$.

Observe that if $\mathfrak{B}$ is asymptotically stable then it is autonomous, i.e. it has no inputs. The following result connects asymptotic stability and QDFs.

Proposition 4. $\mathfrak{B} \in \mathfrak{L}^{\mathrm{w}}$ is asymptotically stable if and only if for every $\Delta \in \mathbb{R}_{S}^{\mathrm{W} \times \mathrm{w}}[\zeta, \eta]$ such that $Q_{\Delta} \stackrel{\mathfrak{B}}{>} 0$ there exists $\Psi \in \mathbb{R}_{S}^{\mathrm{W} \times \mathrm{W}}[\zeta, \eta]$ such that $Q_{\Psi} \stackrel{\mathfrak{B}}{>} 0$ and $\frac{\mathrm{d}}{\mathrm{d} t} Q_{\Psi} \stackrel{\mathfrak{B}}{=}-Q_{\Delta}$.

Proof. This result follows from Theorem 4.8 of [9].

The following definition will be extensively used in this paper.

Definition 5. Let $\mathfrak{B} \in \mathfrak{L}^{\text {w }}$ be asymptotically stable, and $\Psi, \Delta \in$ $\mathbb{R}_{S}^{\mathrm{W} \times \mathrm{W}}[\zeta, \eta]$. A pair of QDFs $\left(Q_{\Psi}, Q_{\Delta}\right)$ is called a Lyapunov pair for $\mathfrak{B}$ if

1. $Q_{\Delta} \stackrel{\mathfrak{B}}{>} 0, Q_{\Psi} \stackrel{\mathfrak{B}}{>} 0$;

2. $\frac{\mathrm{d}}{\mathrm{d} t} Q_{\Psi} \stackrel{\mathfrak{B}}{=}-Q_{\Delta}$.

If $\left(Q_{\Psi}, Q_{\Delta}\right)$ is a Lyapunov pair, then $Q_{\Psi}$ is called a Lyapunov function for $\mathfrak{B}$.

It follows from Proposition 4 that every asymptotically stable behavior $\mathfrak{B} \in \mathfrak{L}^{\mathrm{w}}$ admits a Lyapunov pair. In the following, we also call a "Lyapunov pair" a pair of two-variable polynomial matrices $(\Psi, \Delta)$ inducing a Lyapunov pair $\left(Q_{\Psi}, Q_{\Delta}\right)$ according to Definition 5.

\subsection{Control as interconnection}

The behavior $\mathfrak{B}$ of the full interconnection of a plant $P$ with behavior $\mathfrak{B}_{P}$ and a controller $C$ with behavior $\mathfrak{B}_{C}$ is defined as
$\mathfrak{B}:=\mathfrak{B}_{P} \cap \mathfrak{B}_{C}$ : the plant trajectories are required also to satisfy the laws imposed by the controller. If $\mathfrak{B}_{P}=\operatorname{ker} R_{P}\left(\frac{\mathrm{d}}{\mathrm{d} t}\right), \mathfrak{B}_{C}=$ $\operatorname{ker} R_{C}\left(\frac{\mathrm{d}}{\mathrm{d} t}\right)$, then

$\mathfrak{B}=\mathfrak{B}_{P} \cap \mathfrak{B}_{C}=\operatorname{ker}\left[\begin{array}{c}R_{P}\left(\frac{\mathrm{d}}{\mathrm{d} t}\right) \\ R_{C}\left(\frac{\mathrm{d}}{\mathrm{d} t}\right)\end{array}\right]$.

In this paper, we only consider regular interconnections; in algebraic terms, this is equivalent to the row rank of $\operatorname{col}\left(R_{P}, R_{C}\right)$ being the sum of the row ranks of $R_{P}$ and $R_{C}$, and with $\mathrm{p}\left(\mathfrak{B}_{P} \cap \mathfrak{B}_{C}\right)=$ $\mathrm{p}\left(\mathfrak{B}_{P}\right)+\mathrm{p}\left(\mathfrak{B}_{C}\right)$ in terms of output cardinality (see Section VII of [6]).

\section{Stabilization is dissipation}

Let $\mathfrak{B}_{P} \in \mathfrak{L}^{\mathrm{w}}$ be controllable, and let $\mathfrak{B} \subset \mathfrak{B}_{P}$; then it can be proved (see Theorem 6 of [6]) that there exists $\mathfrak{B}_{C}$ such that the regular full interconnection of $\mathfrak{B}_{P}$ and $\mathfrak{B}_{C}$ satisfies $\mathfrak{B}_{P} \cap \mathfrak{B}_{C}=\mathfrak{B}$; consequently $\mathfrak{B}$ can be described as in (7) for some full row-rank $R_{P}$ and $R_{C}$. In this paper we assume that $\mathfrak{B}$ is asymptotically stable, and consequently autonomous; this implies (see statement 4 of Proposition 1) that $\operatorname{det}\left(\operatorname{col}\left(R_{P}, R_{C}\right)\right) \neq 0$.

The main result of this section is the following.

Theorem 6. Let $\mathfrak{B}_{P} \in \mathfrak{L}^{\mathrm{w}}$ be controllable, and let $\mathfrak{B} \subset \mathfrak{B}_{P}$ be asymptotically stable. Let $\left(Q_{\Psi}, Q_{\Delta}\right)$ be a Lyapunov pair for $\mathfrak{B}$. There exist $Q_{\Psi^{\prime}} \stackrel{\mathfrak{B}}{=} Q_{\Psi}$ and $Q_{\Delta^{\prime}} \stackrel{\mathfrak{B}}{=} Q_{\Delta}$ such that

1. $\mathfrak{B}_{P}$ is $\mathbb{R}^{+}$-half-line dissipative with respect to $\frac{\mathrm{d}}{\mathrm{d} t} Q_{\Psi^{\prime}}+Q_{\Delta^{\prime}}$;

2. $Q_{\Psi^{\prime}} \geq 0$ and $Q_{\Delta^{\prime}} \stackrel{\mathfrak{B}}{>} 0$.

Moreover, let $\mathfrak{B}_{C} \in \mathfrak{L}^{\mathrm{w}}$ be such that the regular full interconnection $\mathfrak{B}_{C} \cap \mathfrak{B}_{P}=\mathfrak{B}$, and let $R_{C} \in \mathbb{R}^{\bullet \times \mathrm{w}}[\xi]$ induce a full row-rank kernel representation of $\mathfrak{B}_{C}$. There exists $Y \in \mathbb{R}^{\mathrm{w} \times \mathrm{w}}[\zeta, \eta]$ such that

$$
\begin{aligned}
& (\zeta+\eta) \Psi^{\prime}(\zeta, \eta)+\Delta^{\prime}(\zeta, \eta) \\
& \stackrel{\mathfrak{B}_{P}}{=} Y(\eta, \zeta)^{\top} R_{C}(\eta)+R_{C}(\zeta)^{\top} Y(\zeta, \eta) .
\end{aligned}
$$

Proof. Let $\mathfrak{B}_{C} \in \mathfrak{L}^{\mathrm{w}}$ be such that the regular full interconnection $\mathfrak{B}_{C} \cap \mathfrak{B}_{P}=\mathfrak{B}$, and let $R_{C} \in \mathbb{R}^{\bullet \times w}[\xi]$ induce a full row-rank kernel representation of $\mathfrak{B}_{C}$. Consider the kernel representation (7) of $\mathfrak{B}$. Conclude from $\frac{\mathrm{d}}{\mathrm{d} t} Q_{\Psi} \stackrel{\mathfrak{B}}{=}-Q_{\Delta}$ and from Proposition 2 that there exists $Y \in \mathbb{R}^{\mathrm{w} \times \mathrm{w}}[\zeta, \eta]$ such that

$$
\begin{aligned}
(\zeta+\eta) \Psi(\zeta, \eta)= & -\Delta(\zeta, \eta)+\left[\begin{array}{l}
R_{P}(\zeta) \\
R_{C}(\zeta)
\end{array}\right]^{\top} Y(\zeta, \eta) \\
& +Y(\eta, \zeta)^{\top}\left[\begin{array}{l}
R_{P}(\eta) \\
R_{C}(\eta)
\end{array}\right] .
\end{aligned}
$$

Now let $\Psi^{\prime}$ and $\Delta^{\prime}$ be the $\operatorname{col}\left(R_{P}, R_{C}\right)$-canonical representatives of $\Psi$ and $\Delta$, respectively. From Proposition 4.10 and Corollary 4.11 of [9], it follows that $\Psi^{\prime}>0$ and $\Delta^{\prime} \geq 0$. Moreover, from $\Delta \stackrel{\mathfrak{B}}{>} 0$ and $\Delta \stackrel{\mathfrak{B}}{=} \Delta^{\prime}$, it also follows that $\Delta^{\prime} \stackrel{\mathfrak{B}}{>} 0$. From $\Psi^{\prime} \stackrel{\mathfrak{B}}{=} \Psi$ and $\Delta^{\prime} \stackrel{\mathfrak{B}}{=} \Delta$, it also follows that $\frac{\mathrm{d}}{\mathrm{d} t} Q_{\Psi}+Q_{\Delta} \stackrel{\mathfrak{B}}{=} \frac{\mathrm{d}}{\mathrm{d} t} Q_{\Psi^{\prime}}+Q_{\Delta^{\prime}}$; consequently, there exists $Y^{\prime} \in \mathbb{R}^{\mathrm{w} \times \mathrm{w}}[\zeta, \eta]$ such that

$$
\begin{aligned}
(\zeta+\eta) \Psi^{\prime}(\zeta, \eta)= & -\Delta^{\prime}(\zeta, \eta)+\left[\begin{array}{l}
R_{P}(\zeta) \\
R_{C}(\zeta)
\end{array}\right]^{\top} Y^{\prime}(\zeta, \eta) \\
& +Y^{\prime}(\eta, \zeta)^{\top}\left[\begin{array}{l}
R_{P}(\eta) \\
R_{C}(\eta)
\end{array}\right]
\end{aligned}
$$


Partition $Y^{\prime}(\zeta, \eta)=\left[\begin{array}{l}Y_{P}^{\prime}(\zeta, \eta) \\ Y_{C}^{\prime}(\zeta, \eta)\end{array}\right]$ corresponding to the partition of $\left[\begin{array}{l}R_{P}(\xi) \\ R_{C}(\xi)\end{array}\right]$, where $Y_{P}^{\prime} \in \mathbb{R}^{\mathrm{p} \times \mathrm{w}}[\zeta, \eta]$ and $Y_{C}^{\prime} \in \mathbb{R}^{(\mathrm{w}-\mathrm{p}) \times \mathrm{w}}[\zeta, \eta]$; then

$$
\begin{aligned}
(\zeta+ & \eta) \Psi^{\prime}(\zeta, \eta)+\Delta^{\prime}(\zeta, \eta)=R_{P}(\zeta)^{\top} Y_{P}^{\prime}(\zeta, \eta)+Y_{P}^{\prime}(\eta, \zeta)^{\top} R_{P}(\eta) \\
& +R_{C}(\zeta)^{\top} Y_{C}^{\prime}(\zeta, \eta)+Y_{C}^{\prime}(\eta, \zeta)^{\top} R_{C}(\eta) .
\end{aligned}
$$

Now define $\Sigma(\zeta, \eta):=R_{C}(\zeta)^{\top} Y_{C}^{\prime}(\zeta, \eta)+Y_{C}^{\prime}(\eta, \zeta)^{\top} R_{C}(\eta)$; it follows from (9) that $\frac{\mathrm{d}}{\mathrm{d} t} Q_{\Psi^{\prime}}+Q_{\Delta^{\prime}} \stackrel{\mathfrak{B}_{P}}{=} Q_{\Sigma}$. From this equality, it follows that $\int_{-\infty}^{+\infty} Q_{\Sigma}(w) \mathrm{d} t=\int_{-\infty}^{+\infty} Q_{\Delta^{\prime}}(w) \mathrm{d} t$ for all $w \in$ $\mathfrak{B}_{P} \cap \mathfrak{D}\left(\mathbb{R}, \mathbb{R}^{\mathrm{W}}\right)$; moreover, since $\Delta^{\prime} \geq 0$, it follows that $Q_{\Delta^{\prime}}$ is a dissipation function and that $\mathfrak{B}_{P}$ is $\Sigma$-dissipative. From the fact that the storage function $Q_{\psi^{\prime}}>0$ and from Theorem 6.3 of [9], it follows that $\mathfrak{B}_{P}$ is $\mathbb{R}^{+}$-half-line dissipative with respect to $Q_{\Sigma}$. This concludes the proof of the first part of the theorem.

The second part of the theorem follows easily from the argument used in the proof of the first part. This concludes the proof.

Remark 7. Theorem 6 shows that interconnecting a plant $\mathfrak{B}_{P}$ with a stabilizing controller $\mathfrak{B}_{C}$ in a regular and full interconnection imposes on the plant the requirement that it be $\mathbb{R}^{+}$-half-line dissipative with respect to a supply rate $Q_{\Sigma}$ respectively associated with a Lyapunov pair $(\Psi, \Delta)$ and to the stabilizing controller as follows. We can define $Q_{\Sigma}:=\frac{\mathrm{d}}{\mathrm{d} t} Q_{\Psi}+Q_{\Delta}$, i.e. $Q_{\Psi}$ to be the storage function and $Q_{\Delta}$ to be the dissipation function for $Q_{\Sigma}$; or we can define $Q_{\Sigma}$ to be the QDF induced by the two-variable polynomial matrix on the right-hand side of (8), where the representation of the controller is explicitly present. These two QDFs are $\mathfrak{B}_{P^{-}}$ equivalent, and consequently for both of them Eq. (6) holds. Note that $Q_{\Sigma}$ is in general a dynamic, not a static, supply rate.

Remark 8. Stabilizing a plant using energy methods is a wellknown technique in the nonlinear setting; see [1-4]. The first part of Theorem 6 shows that every stabilizing controller can be interpreted as imposing dissipation on the plant. In the linear case, stabilization is always dissipation.

Remark 9. The relation between the supply rate associated with a Lyapunov pair and a controller $\mathfrak{B}_{C}$ yielding an asymptotically stable closed-loop behavior $\mathfrak{B}$ is rather loose. On the one hand, given $\mathfrak{B}_{C}$, there are many Lyapunov pairs for $\mathfrak{B}$ and consequently many supply rates; on the other hand, given a Lyapunov pair for $\mathfrak{B}$, for any controller $C$ yielding $\mathfrak{B}$ there exists some $Y \in \mathbb{R}^{\mathrm{w} \times \mathrm{w}}[\zeta, \eta]$ satisfying (8).

The following result - which we state without proof because of lack of space - consists for the first part of Kuijper's parameterization of stabilizing controllers (see Theorem 3.3 of [11]). The second part shows how a supply rate can be expressed in terms of the parameters of a controller $C$ yielding $\mathfrak{B}$.

Proposition 10. Let (7) and

$$
\begin{aligned}
& w=M\left(\frac{\mathrm{d}}{\mathrm{d} t}\right) \ell \\
& 0=D\left(\frac{\mathrm{d}}{\mathrm{d} t}\right) \ell
\end{aligned}
$$

be a minimal kernel, respectively hybrid representation of an autonomous asymptotically stable subbehavior $\mathfrak{B}$ of $\mathfrak{B}_{P}=\operatorname{ker} R_{P}$ $\left(\frac{\mathrm{d}}{\mathrm{d} t}\right)$. Then there exist $C_{0} \in \mathbb{R}^{\mathrm{m}(\mathfrak{B}) \times \mathrm{w}}[\xi]$ and $N \in \mathbb{R}^{\mathrm{w} \times \mathrm{p}(\mathfrak{B})}[\xi]$ such that

$\left[\begin{array}{l}R_{P} \\ C_{0}\end{array}\right]\left[\begin{array}{ll}N & M\end{array}\right]=\left[\begin{array}{cc}I_{\mathrm{p}(\mathfrak{B})} & 0 \\ 0 & I_{\mathrm{m}(\mathfrak{B})}\end{array}\right]$.
Moreover, $\mathfrak{B}_{C}=\operatorname{ker} R_{C}\left(\frac{\mathrm{d}}{\mathrm{d} t}\right) \in \mathfrak{L}^{\mathrm{w}}$ satisfies $\mathfrak{B}_{P} \cap \mathfrak{B}_{C}=\mathfrak{B}$ if and only if there exist $G \in \mathbb{R}^{\mathrm{m}(\mathfrak{B}) \times \mathrm{p}(\mathfrak{B})}[\xi]$ and $U \in \mathbb{R}^{\mathrm{m}(\mathfrak{B}) \times \mathrm{m}(\mathfrak{B})}[\xi]$ unimodular, such that

$R_{C}=G R_{P}+U D C_{0}$.

Let $G, U$, and $D$ be matrices such that (10) holds. Let $\left(\Psi^{\prime}, \Delta^{\prime}\right)$ be a $\operatorname{col}\left(R_{P}, R_{C}\right)$-canonical Lyapunov pair for $\mathfrak{B}$. Then there exist matrices $Y_{P}, Y_{C} \in \mathbb{R}^{\bullet \times \bullet}[\xi]$ such that

$$
\begin{aligned}
& (\zeta+\eta) \Psi^{\prime}(\zeta, \eta)+\Delta^{\prime}(\zeta, \eta) \\
& \quad \stackrel{\mathfrak{B}_{P}}{=} Y_{C}(\zeta)^{\top}\left(G(\eta) R_{P}(\eta)+U(\eta) D(\eta) C_{0}(\eta)\right) \\
& \quad+\left(G(\zeta) R_{P}(\zeta)+U(\zeta) D(\zeta) C_{0}(\zeta)\right)^{\top} Y_{C}(\eta)
\end{aligned}
$$

Using this result, one can show how one supply rate expressed in terms of one controller yielding $\mathfrak{B}$ can be expressed in terms of any other controller yielding the same closed-loop behavior. We do not enter into these details.

\subsection{The state-space case}

In order to illustrate the result of Theorem 6, in this section we consider the specialization of our results to the case of state-space systems.

We first consider the case of a controllable plant $\frac{\mathrm{d}}{\mathrm{d} t} x=A x+B u$ stabilized by static feedback $u=-K x$. The set of all $x$-trajectories for which there exists a $u$ satisfying these equations is described by the usual closed-loop state equations $\frac{\mathrm{d}}{\mathrm{d} t} x=(A-B K) x$.

Now let $(P, D)$ be a Lyapunov pair for the closed-loop behavior, i.e. $P>0, D>0$, and $\frac{\mathrm{d}}{\mathrm{d} t} x^{\top} P x=-x^{\top} D x$ for all $x$ satisfying $\frac{\mathrm{d}}{\mathrm{d} t} x=(A-B K) x$. Since the state variable $x$ is minimal, this is equivalent to $(A-B K)^{\top} P+(A-B K) P=-D$.

Consider now the quadratic form $Q_{\Phi}$ acting on the trajectories $\operatorname{col}(x, u)$ of the plant defined by

$$
\begin{aligned}
Q_{\Phi}(x, u) & :=\left[\begin{array}{ll}
x^{\top} & u^{\top}
\end{array}\right]\left[\begin{array}{cc}
K^{\top} B^{\top} P+P B K & P B \\
B^{\top} P & 0
\end{array}\right]\left[\begin{array}{l}
x \\
u
\end{array}\right] \\
& =x^{\top}\left(K^{\top} B^{\top} P+P B K\right) x+u^{\top} B^{\top} P x+x^{\top} P B u .
\end{aligned}
$$

Observe that along the trajectories of the plant $B u=\frac{\mathrm{d}}{\mathrm{d} t} x-A x$, and consequently

$$
\begin{aligned}
Q_{\Phi}(x, u)= & x^{\top}\left(K^{\top} B^{\top} P+P B K\right) x \\
& +\left(\frac{\mathrm{d}}{\mathrm{d} t} x-A x\right)^{\top} P x+x^{\top} P\left(\frac{\mathrm{d}}{\mathrm{d} t} x-A x\right) x .
\end{aligned}
$$

From this last expression, straightforward manipulations yield that, for all trajectories $\operatorname{col}(x, u)$ satisfying $\frac{\mathrm{d}}{\mathrm{d} t} x=A x+B u$, it holds that

$$
\begin{aligned}
Q_{\Phi}(x, u)= & \frac{\mathrm{d}}{\mathrm{d} t}\left(x^{\top} P x\right)+x^{\top}\left(K^{\top} B^{\top}-A^{\top}\right) P x \\
& +x^{\top} P(B K-A) x \\
= & \frac{\mathrm{d}}{\mathrm{d} t}\left(x^{\top} P x\right)+x^{\top} D x,
\end{aligned}
$$

where we have used the fact that $(A-B K)^{\top} P+(A-B K) P=-D$. It follows that the plant behavior is dissipative with respect to the supply rate $Q_{\Phi}$; moreover, since the storage function $P$ is positive definite, it follows that the plant is in fact $\mathbb{R}_{+}$-half-line dissipative.

To see how the computations sketched previously fit within the result of Theorem 6 , define the variable $w:=\operatorname{col}(x, u)$, and observe that the plant behavior $\mathfrak{B}_{P}$ is represented in kernel form by $R_{P}(\xi):=\left[\begin{array}{ll}\xi I_{\mathrm{n}}-A & -B\end{array}\right]$, and that the controller behavior $\mathfrak{B}_{C}$ is 
represented in kernel form by $R_{C}(\xi):=\left[\begin{array}{ll}K & I_{\mathrm{m}}\end{array}\right]$. The closed-loop behavior $\mathfrak{B}=\mathfrak{B}_{P} \cap \mathfrak{B}_{C}$ has the kernel representation induced by

$R(\xi):=\operatorname{col}\left(R_{P}(\xi), R_{C}(\xi)\right)=\left[\begin{array}{cc}\xi I_{\mathrm{n}}-A & -B \\ K & I_{\mathrm{m}}\end{array}\right]$.

Now, using Lemma $6.3-11$ of [12] and the definition of $R$ canonical representative, it is straightforward to see that $X \in$ $\mathbb{R}_{S}^{(\mathrm{n}+\mathrm{m}) \times(\mathrm{n}+\mathrm{m})}[\zeta, \eta]$ is $R$-canonical if and only if it is of the form $X=\left[\begin{array}{cc}X_{1} & 0 \\ 0 & 0\end{array}\right]$, where $X_{1} \in \mathbb{R}_{s}^{\mathrm{n} \times \mathrm{n}}$. Consequently, the $R$-canonical Lyapunov pair is of the form

$\Psi:=\left[\begin{array}{ll}P & 0 \\ 0 & 0\end{array}\right]$ and $\Delta:=\left[\begin{array}{ll}D & 0 \\ 0 & 0\end{array}\right]$,

for some $P>0, D>0$. The matrix $Y^{\prime}$ of the proof of Theorem 6 is

$Y^{\prime}:=\left[\begin{array}{cc}P & 0 \\ B^{\top} P & 0\end{array}\right]$

it is straightforward to verify that it satisfies

$(\zeta+\eta)\left[\begin{array}{ll}P & 0 \\ 0 & 0\end{array}\right]=-\left[\begin{array}{ll}D & 0 \\ 0 & 0\end{array}\right]+R(\zeta)^{\top} Y^{\prime}(\eta)+Y^{\prime}(\zeta)^{\top} R(\eta)$.

Moreover, the matrix on the right-hand side of Eq. (8) is

$\left[\begin{array}{c}K^{\top} \\ I_{\mathrm{m}}\end{array}\right]\left[\begin{array}{ll}B^{\top} P & 0\end{array}\right]+\left[\begin{array}{c}P B \\ 0\end{array}\right]\left[\begin{array}{ll}K & I_{\mathrm{m}}\end{array}\right]$,

i.e., the matrix inducing the quadratic form $Q_{\Phi}$ defined in (11).

We now consider the case of the (regular, full) interconnection of a state-space plant $\frac{\mathrm{d}}{\mathrm{d} t} x_{1}=A_{1} x+B_{1} u_{1}$ with trajectories $\operatorname{col}\left(x_{1}, u_{1}\right)$ and of a dynamic controller $\frac{\mathrm{d}}{\mathrm{d} t} x_{2}=A_{2} x+B_{2} u_{2}$ with trajectories $\operatorname{col}\left(x_{2}, u_{2}\right)$ under the interconnection constraints $u_{2}=x_{1}, u_{1}=x_{2}$. Denote the dimensions of the (minimal) state variables $x_{i}$ with $n_{i}, i=1,2$. The closed-loop behavior is described by the equations

$\frac{\mathrm{d}}{\mathrm{d} t}\left[\begin{array}{l}x_{1} \\ x_{2}\end{array}\right]=\left[\begin{array}{ll}A_{1} & B_{1} \\ B_{2} & A_{2}\end{array}\right]\left[\begin{array}{l}x_{1} \\ x_{2}\end{array}\right]$.

Let $P=\left[\begin{array}{ll}P_{11} & P_{12} \\ P_{12}^{\top} & P_{22}\end{array}\right]$ induce a Lyapunov function for the closed-loop behavior; i.e. $\frac{\mathrm{d}}{\mathrm{d} t}\left[\begin{array}{ll}x_{1}^{\top} & x_{2}^{\top}\end{array}\right] P\left[\begin{array}{l}x_{1} \\ x_{2}\end{array}\right]=-\left[\begin{array}{ll}x_{1}^{\top} & x_{2}^{\top}\end{array}\right] D\left[\begin{array}{l}x_{1} \\ x_{2}\end{array}\right]$ along the trajectories of the closed-loop behavior, with $D=\left[\begin{array}{ll}D_{11} & D_{12} \\ D_{12}^{\top} & D_{22}\end{array}\right]>0$; equivalently, in view of the minimality of the state variables,

$$
\begin{gathered}
{\left[\begin{array}{ll}
A_{1} & B_{1} \\
B_{2} & A_{2}
\end{array}\right]^{\top}\left[\begin{array}{ll}
P_{11} & P_{12} \\
P_{12}^{\top} & P_{22}
\end{array}\right]} \\
+\left[\begin{array}{ll}
P_{11} & P_{12} \\
P_{12}^{\top} & P_{22}
\end{array}\right]\left[\begin{array}{ll}
A_{1} & B_{1} \\
B_{2} & A_{2}
\end{array}\right]=-\left[\begin{array}{ll}
D_{11} & D_{12} \\
D_{12}^{\top} & D_{22}
\end{array}\right] .
\end{gathered}
$$

Now define the following quadratic functional of the plant variables and their derivatives:

$Q_{\Phi}\left(x_{1}, u_{1}\right):=2\left(x_{1}^{\top} P_{12}+u_{1}^{\top} P_{22}\right)\left(-B_{2} x_{1}+\frac{\mathrm{d}}{\mathrm{d} t} u_{1}-A_{2} u_{1}\right) ;$

observe that $Q_{\Phi}$ arises from a combination of the controller dynamics and of the Lyapunov matrix $P$, and that it also involves derivatives of the input variable $u_{1}$. Use (13) to check that

$$
\begin{aligned}
- & {\left[\begin{array}{c}
B_{2}^{\top} \\
A_{2}^{\top}
\end{array}\right]\left[\begin{array}{ll}
P_{12}^{\top} & P_{22}
\end{array}\right]-\left[\begin{array}{l}
P_{12} \\
P_{22}
\end{array}\right]\left[\begin{array}{ll}
B_{2} & A_{2}
\end{array}\right] } \\
= & {\left[\begin{array}{ll}
D_{11} & D_{12} \\
D_{12}^{\top} & D_{22}
\end{array}\right]+\left[\begin{array}{l}
A_{1}^{\top} \\
B_{1}^{\top}
\end{array}\right]\left[\begin{array}{ll}
P_{11} & P_{12}
\end{array}\right] } \\
& +\left[\begin{array}{l}
P_{11} \\
P_{12}^{\top}
\end{array}\right]\left[\begin{array}{ll}
A_{1} & B_{1}
\end{array}\right] .
\end{aligned}
$$

Use the last equality and the fact that along the trajectories of the plant $A_{1} x_{1}+B_{1} u_{1}=\frac{\mathrm{d}}{\mathrm{d} t} x_{1}$ to verify that

$Q_{\Phi}\left(x_{1}, u_{1}\right)=\frac{\mathrm{d}}{\mathrm{d} t}\left(\left[\begin{array}{ll}x_{1}^{\top} & u_{1}^{\top}\end{array}\right] P\left[\begin{array}{l}x_{1} \\ u_{1}\end{array}\right]\right)+\left[\begin{array}{ll}x_{1}^{\top} & u_{1}^{\top}\end{array}\right] D\left[\begin{array}{l}x_{1} \\ u_{1}\end{array}\right] ;$

now conclude from $P, D>0$ that the plant is dissipative with respect to $Q_{\Phi}$.

To see how these computations fit within the result of Theorem 6, observe that the closed-loop behavior is represented in kernel form by the matrix

$\left[\begin{array}{l}R_{P}(\xi) \\ R_{C}(\xi)\end{array}\right]:=\left[\begin{array}{cc}\xi I_{n_{1}}-A_{1} & -B_{1} \\ -B_{2} & \xi I_{n_{2}}-A_{2}\end{array}\right]$.

It is a matter of immediate verification using the definition of $R$ canonical representative and Lemma 6.3-11 of [12] to see that $\operatorname{col}\left(R_{P}, R_{C}\right)$-canonical Lyapunov pairs are induced by constant matrices. Let $P$ and $D$ be a Lyapunov pair; then Theorem 6 states that the plant is dissipative with respect to the supply rate induced by

$$
\left[\begin{array}{c}
-B_{2}^{\top} \\
\zeta I_{n_{2}}-A_{2}^{\top}
\end{array}\right]\left[\begin{array}{ll}
P_{12}^{\top} & P_{22}
\end{array}\right]+\left[\begin{array}{l}
P_{12} \\
P_{22}
\end{array}\right]\left[\begin{array}{ll}
-B_{2} & \eta I_{n_{2}}-A_{2}
\end{array}\right],
$$

and that along the trajectories of the plant this supply rate is equivalent to that induced by the two-variable polynomial matrix $(\zeta+\eta) P+D$. Observe that this supply rate acting on the plant variables is precisely the $\mathrm{QDF} Q_{\Phi}$ defined in (14).

\section{Stable interconnections and dissipation}

The main result of this section is a theorem showing that the regular, full interconnection of two linear, time-invariant systems $\mathfrak{B}_{1}$ and $\mathfrak{B}_{2}$ is stable if and only if there exists a QDF $Q_{\Phi}$ such that $\mathfrak{B}_{1}$ and $\mathfrak{B}_{2}$ are dissipative with respect to the supply rate $Q_{\Phi}$ and $-Q_{\Phi}$, respectively.

Theorem 11. Let $\mathfrak{B}_{i} \in \mathfrak{L}^{\mathrm{w}}$ be controllable behaviors, $i=1$, 2. The following statements are equivalent.

1. The regular full interconnection of $\mathfrak{B}_{1}$ and $\mathfrak{B}_{2}$ is asymptotically stable.

2. There exist $Q D F s Q_{\Phi}, Q_{\Psi} \geq 0$ and $Q_{\Delta} \geq 0$ with the following properties:

(2.a) $\mathfrak{B}_{1}$ is dissipative with respect to $Q_{\Phi} \stackrel{\mathfrak{B}_{1}}{=} \frac{\mathrm{d}}{\mathrm{d} t} Q_{\Psi}+Q_{\Delta}$, and $\mathfrak{B}_{2}$ is dissipative with respect to $-Q_{\Phi} \stackrel{\mathfrak{B}_{2}}{=} \frac{\mathrm{d}}{\mathrm{d} t} Q_{\Psi}+Q_{\Delta}$;

(2.b) $Q_{\Delta} \stackrel{\mathfrak{B}_{1} \cap \mathfrak{B}_{2}}{>} 0$.

3. There exists a supply rate $Q_{\Phi}$ with the following properties:

(3.a) $\mathfrak{B}_{1}$ is $\mathbb{R}^{+}$-half-line dissipative with respect to $Q_{\Phi}$, and $\mathfrak{B}_{2}$ is $\mathbb{R}^{+}$-half-line dissipative with respect to $-Q_{\Phi}$;

(3.b) The dissipation function $Q_{\Delta}$ satisfies $Q_{\Delta} \stackrel{\mathfrak{B}_{1} \cap \mathfrak{B}_{2}}{>} 0$.

Proof. We begin by proving the equivalence of statements (1) and (2).

$(1) \Longrightarrow(2)$ : Let $R_{i} \in \mathbb{R}^{\mathrm{p}\left(\mathfrak{B}_{i}\right) \times \mathrm{w}}[\xi], i=1,2$, induce full rowrank kernel representations of $\mathfrak{B}_{i}$, and define $R:=\operatorname{col}\left(R_{1}, R_{2}\right)$. Since $\mathfrak{B}_{1} \cap \mathfrak{B}_{2}$ is asymptotically stable, for every $R$-canonical $\Delta \in$ $\mathbb{R}_{s}^{\mathrm{W} \times \mathrm{W}}[\zeta, \eta]$ the two-variable polynomial Lyapunov equation

$(\zeta+\eta) \Psi(\zeta, \eta)=-\Delta(\zeta, \eta)+R(\zeta)^{\top} Y(\eta)+Y(\zeta)^{\top} R(\eta)$

has an $R$-canonical solution $\Psi \in \mathbb{R}_{S}^{\mathrm{W} \times \mathrm{w}}[\zeta, \eta]$ (see Proposition 4.4 of [13]). Now choose $\Delta$ such that $Q_{\Delta} \geq 0$ and $Q_{\Delta} \stackrel{\mathfrak{B}_{1} \cap \mathfrak{B}_{2}}{>} 0$; from Theorem 4.12 of [9] it follows that $Q_{\psi} \geq 0$. Now rewrite the above equation as

$$
\begin{aligned}
(\zeta+\eta) \Psi(\zeta, \eta)+\Delta(\zeta, \eta)= & R(\zeta)^{\top} Y(\eta)+Y(\zeta)^{\top} R(\eta) \\
= & R_{1}(\zeta)^{\top} Y_{1}(\eta)+Y_{1}(\zeta)^{\top} R_{1}(\eta) \\
& +R_{2}(\zeta)^{\top} Y_{2}(\eta)+Y_{2}(\zeta)^{\top} R_{2}(\eta),
\end{aligned}
$$

where $Y_{i} \in \mathbb{R}^{\mathrm{p}\left(\mathfrak{B}_{i}\right) \times \mathrm{w}}[\xi], i=1,2$, are defined from a partition of $Y$ compatible with the partition of $R$. From the last equality, it follows that 


$$
\begin{aligned}
(\zeta+ & \eta) \frac{1}{2} \Psi(\zeta, \eta)+\frac{1}{2} \Delta(\zeta, \eta)-\left(R_{1}(\zeta)^{\top} Y_{1}(\eta)+Y_{1}(\zeta)^{\top} R_{1}(\eta)\right) \\
= & (\zeta+\eta)\left(-\frac{1}{2} \Psi(\zeta, \eta)\right)-\frac{1}{2} \Delta(\zeta, \eta) \\
& +R_{2}(\zeta)^{\top} Y_{2}(\eta)+Y_{2}(\zeta)^{\top} R_{2}(\eta) .
\end{aligned}
$$

Now define

$$
\begin{aligned}
\Phi(\zeta, \eta):= & (\zeta+\eta)\left(\frac{1}{2} \Psi(\zeta, \eta)\right) \\
& +\frac{1}{2} \Delta(\zeta, \eta)-R_{1}(\zeta)^{\top} Y_{1}(\eta)-Y_{1}(\zeta)^{\top} R_{1}(\eta)
\end{aligned}
$$

and observe that along $\mathfrak{B}_{1}=\operatorname{ker} R_{1}\left(\frac{\mathrm{d}}{\mathrm{d} t}\right)$ it holds that $Q_{\Phi} \stackrel{\mathfrak{B}_{1}}{=} \frac{\mathrm{d}}{\mathrm{d} t}$ $\left(\frac{1}{2} Q_{\Psi}\right)+\frac{1}{2} Q_{\Delta}$. Also, along $\mathfrak{B}_{2}=\operatorname{ker} R_{2}\left(\frac{\mathrm{d}}{\mathrm{d} t}\right)$ it holds that $Q_{-\Phi} \stackrel{\mathfrak{B}_{2}}{=}$ $\frac{\mathrm{d}}{\mathrm{d} t}\left(\frac{1}{2} Q_{\Psi}\right)+\frac{1}{2} Q_{\Delta}$. This concludes the proof of $(1) \Longrightarrow(2)$.

$(2) \Longrightarrow(1)$ : Consider that under assumptions (2.a) there exist polynomial matrices $Y_{i} \in \mathbb{R}^{\mathrm{p}\left(\mathfrak{B}_{i}\right) \times \mathrm{w}}[\xi], i=1$, 2, such that

$$
\begin{aligned}
\Phi(\zeta, \eta)= & (\zeta+\eta) \Psi(\zeta, \eta)+\Delta(\zeta, \eta)+R_{1}(\zeta)^{\top} Y_{1}(\zeta, \eta) \\
& +Y_{1}(\zeta, \eta)^{\top} R_{1}(\eta) \\
-\Phi(\zeta, \eta)= & (\zeta+\eta) \Psi(\zeta, \eta)+\Delta(\zeta, \eta)+R_{2}(\zeta)^{\top} Y_{2}(\zeta, \eta) \\
& +Y_{2}(\eta, \zeta)^{\top} R_{2}(\eta) .
\end{aligned}
$$

Adding the two equations and rearranging, we obtain

$$
\begin{aligned}
-\left(R(\zeta)^{\top} Y(\zeta, \eta)+Y(\eta, \zeta)^{\top} R(\eta)\right)= & (\zeta+\eta) 2 \Psi(\zeta, \eta) \\
& +2 \Delta(\zeta, \eta),
\end{aligned}
$$

where $R:=\operatorname{col}\left(R_{1}, R_{2}\right)$ and $Y:=\operatorname{col}\left(Y_{1}, Y_{2}\right)$. From (2.b) and $\Psi \geq 0$, it follows that $Q_{\Psi}$ is a Lyapunov function for ker $R\left(\frac{\mathrm{d}}{\mathrm{d} t}\right)=\mathfrak{B}_{1} \cap \mathfrak{B}_{2}$. This concludes the proof of the equivalence of (1) and (2).

In order to prove the equivalence of statements (2) and (3), recall from Theorem 6.3 of [9] that half-line dissipativity is equivalent to the existence of a nonnegative storage function.

Remark 12. The result of Proposition 1, p. 438, of [5] can be translated into our notation as follows. Let $\mathfrak{B}_{P}$ and $\mathfrak{B}_{C}$ be controllable behaviors, and assume that $\mathfrak{B}_{P}$ is dissipative with respect to a $\mathrm{QDF} Q_{\Phi}$ with nonnegative storage function, and that $\mathfrak{B}_{C}$ is strictly dissipative with respect to $-Q_{\Phi}$, i.e., there exists $\epsilon>0$ such that $\int_{-\infty}^{0} Q_{\Phi}(w)(t) \mathrm{d} t \geq \epsilon \int_{-\infty}^{0}\|w(t)\|^{2} \mathrm{~d} t$ for all infinitely differentiable $w \in \mathfrak{B}$ of compact support; then the interconnection $\mathfrak{B}_{P} \cap \mathfrak{B}_{C}$ is asymptotically stable. The result of Theorem 11 provides a necessary condition for asymptotic stability of the interconnection; moreover, the proof shows how the supply rate $Q_{\Phi}$ is related to a Lyapunov pair for the interconnected system.

\subsection{The state-space case}

We now consider the application of Theorem 11 to controllable state-space systems $\frac{\mathrm{d}}{\mathrm{d} t} x=A x+B u$ and constant state-feedback controllers $u=-K x$. Consider first the case in which the interconnected system is asymptotically stable, and let $P=P^{\top}>$ 0 and $D=D^{\top}>0$ be a Lyapunov pair. Define the following quadratic functional acting on infinitely differentiable trajectories $\operatorname{col}(x, u)$ :

$Q_{\Phi}(x, u)=\frac{1}{2} \frac{\mathrm{d}}{\mathrm{d} t}\left(x^{\top} P x\right)+\frac{1}{2} x^{\top} D x-2 x^{\top} P\left(\frac{\mathrm{d}}{\mathrm{d} t} x-A x-B u\right)$,

and observe that, since $P$ and $D$ form a Lyapunov pair, i.e., $\frac{1}{2} \frac{\mathrm{d}}{\mathrm{d} t}\left(x^{\top} P x\right)+\frac{1}{2} x^{\top} D x=0$ along the trajectories of the interconnected system, it also holds that

$Q_{\Phi}(x, u)=-\frac{1}{2} \frac{\mathrm{d}}{\mathrm{d} t}\left(x^{\top} P x\right)-\frac{1}{2} x^{\top} D x+2 x^{\top} P B^{\top}(K x+u)$.

It is straightforward to check that the set of trajectories satisfying $\frac{\mathrm{d}}{\mathrm{d} t} x=A x+B u$ is dissipative with respect to $Q_{\Phi}$, while the set of trajectories of the controller $u=-K x$ is dissipative with respect to $-Q_{\phi}$; indeed, along these trajectories the functional $Q_{\Phi}$, respectively $-Q_{\Phi}$, equal $\frac{1}{2} \frac{\mathrm{d}}{\mathrm{d} t}\left(x^{\top} P x\right)+\frac{1}{2} x^{\top} D x$. Observe that the dissipation function $x^{\top} D x$ for the plant is a function of the state only, and does not involve the input, as happens in general (see Theorem 6.2 of [10]); moreover, the controller has a nonzero storage function even if it is memoryless. The implication (1) $\Longrightarrow$ (2) of the theorem is thus verified; the converse implication is essentially a restatement of the well-known passivity theorem.

\section{Conclusions}

Theorem 6 states that stabilization by regular full interconnection is equivalent to the controller imposing dissipation on the trajectories of the plant, and that the supply rate imposed by the controller is associated with a Lyapunov pair for the closed-loop behavior. Theorem 11 states that the interconnection of two systems is stable if and only if one of them is half-line dissipative with respect to a supply rate $Q_{\Phi}$, and the other with respect to $-Q_{\Phi}$. Current research efforts are aimed at deriving analogous results for the case of linear distributed systems, and at investigating their use for the computation of stabilizing controllers.

\section{Acknowledgements}

The authors would like to express their gratitude to Prof. Eva Zerz and Prof. Jan C. Willems for fruitful discussions and precious advice, and to the referees, whose suggestions contributed greatly to improving the readability of the paper.

\section{References}

[1] A.M. Bloch, N.E. Leonard, J.E. Marsden, Controlled Lagrangians and the stabilization of mechanical systems I: the first matching theorem, IEEE Trans. Syst. Control 45 (2000) 2253-2270.

[2] A.M. Bloch, D.-E. Chang, N.E. Leonard, J.E. Marsden, Controlled Lagrangians and the stabilization of mechanical systems II: potential shaping, IEEE Trans. Automat. Control 46 (2001) 1556-1571.

[3] B. Maschke, R. Ortega, A. van der Schaft, Energy-based Lyapunov functions for forced Hamiltonian systems with dissipation, IEEE Trans. Automat. Control 45 (2001) 1498-1502.

[4] R. Ortega, M.W. Spong, F. Gómez-Estern, G. Blankenstein, Stabilization of a class of underactuated mechanical systems via interconnection and damping assignment, IEEE Trans. Automat. Control 47 (2002) 1218-1233.

[5] K. Takaba, Robust stability analysis of uncertain interconnections, SICE J. Control Meas. Syst. Int. 1 (6) (2008) 435-442.

[6] J.C. Willems, On interconnections, control, and feedback, IEEE Trans. Automat. Control 42 (1997) 326-339.

[7] P. Rapisarda, J.C. Willems, State maps for linear systems, SIAM J. Control Optim. 35 (3) (1997) 1053-1091.

[8] J.W. Polderman, J.C. Willems, Introduction to Mathematical System Theory: A Behavioral Approach, Springer-Verlag, Berlin, 1997.

[9] J.C. Willems, H.L. Trentelman, On quadratic differential forms, SIAM J. Control Optim. 36 (5) (1998) 1703-1749.

[10] H.L. Trentelman, J.C. Willems, Every storage function is a state function, Syst. Control Lett. 32 (1997) 249-259.

[11] M. Kuijper, Why do stabilizing controllers stabilize? Automatica 31 (1995) 621-625.

[12] T. Kailath, Linear Systems, Prentice-Hall, Englewood Cliffs, NJ, 1980.

[13] R. Peeters, P. Rapisarda, A two-variable approach to solve the polynomial Lyapunov equation, Syst. Control Lett. 42 (2001) 117-126. 